\title{
Study on Interacting Multiple Model-Unscented Filter Algorithm for Airspace Maneuvering Target in Radar Networking*
}

\author{
ZHAO Wen-bo, DING Hai-long, DU Ji-yan \\ New Star Research Institute of Applied Technology in Hefei City, Anhui Province 230031, China
}

\begin{abstract}
Using radar networking system (RNS) to track airspace target, the maneuvering target model is uncertain and the system is nonlinear. Constructed-parameter interacting mutiple model-unscented filter algorithm (IMM-UFA) proposed in this paper, by means of modeling initialization based on original points of flighting path, solves the problem of state estimation for tracking airspace target in RNS. The simulating verification demonstrates that constructed-parameter IMM-UFA proposed in this paper has high precision of state estimation and strong adaptability in tracking maneuvering target and good usability engineering.
\end{abstract}

Index Terms - radar networking, interacting multiple model, unscented filter, initialization

\section{Introduction}

Radar networking system (RNS) ${ }^{[1]}$ is indeed multi-source data fusion processing system. Its data sensors are radars allocated at different places according to optimal embattling principle. Interacting multiple model algorithm (IMMA) ${ }^{[2,3]}$ bases on filtering algorithm, with multi-model at core. KFA is a optimal estimation algorithm in linear Gaussian system ${ }^{[4,5]}$. In RNS the observation data transmitted to fusion center is in form of rang, azimuth, and elevation angle (or altitude) in radar pole coordinate system. However, target state is estimated in fusion center rectangular coordinate system. As a result, the observation data has nonlinear relationship with state value. The nonlinear relation does not satisfy linear requirement of KFA application. Based on unscented transform(UT), UFA ${ }^{[6,7]}$ approximates the probability distribution of the nonlinear state/observation equation through particle collector (Sigma point set), which is deterministically chosen based on the principle of statistical convergence. UFA, formed through a combination of UT and linear minimum variance estimation algorithm. This paper intends to bind IMMA and UFA (IMM-UFA), which solve problem of airspace maneuvering target filtering estimation in RNS through analyzing the relevant parameter modeling.

\section{Airspace Maneuvering Target State Linear Minimum Variance Estimation in RNS}

Let $\mathrm{X}$ and $\mathrm{Z}$ be true state and detect data of airspace maneuvering target $\mathrm{T}$ at timestep $\mathrm{k}$ in RNS. The pairing state solution set of minimum variance estimation is ${ }^{[8]}$.

$$
J=\left.E_{x, Z}\left\{[X-\hat{X}(Z)]^{T}[X-\hat{X}(Z)]\right\}\right|_{\hat{X}(Z)=\hat{x}_{x \in c}(Z)}=\min
$$

When $\mathrm{X}$ and $\mathrm{Z}$ were distributed Gaussian white noise, Calculating the state estimation and its covariance ${ }^{[8]}$

$$
\begin{gathered}
\hat{X}_{x y}(Z)=m_{x}+P_{x z} P_{z}^{-1}\left(Z-m_{z}\right) \\
P=P_{x}-P_{x z} P_{z}^{-1} P_{z x}
\end{gathered}
$$

Where $m_{x}, m_{z}, P_{x}, P_{x z}$ are mean of state, mean of measurement value, state covariance matrix, state-measuring cross-covariance matrix. If we can get these parameters, we could resolve linear minimum variance estimation of airspace target $\mathrm{T}$.

\section{Statistics Characterization of Target State/Measurement}

This paper use UT to obtain the required four statistical parameters for the linear minimum variance estimation in the process of airspace maneuvering target state estimation in RNS. According to the references [6,7], based on the scaled sampling, objective state $\sigma$ point set and their corresponding weights at timestep k-1 are calculated as formula (4) and (5) below

$$
\begin{aligned}
& \left\{\begin{array}{l}
\chi_{k-1}^{0}=\hat{X}_{k-1} \quad \mathrm{~h}=0 \\
\chi_{k-1}^{h}=\hat{X}_{k-1}+\left(\sqrt{(n+\lambda) P_{k-1}}\right)_{h} \mathrm{~h}=1, \cdots, n \\
\chi_{k-1}^{h}=\hat{X}_{k-1}-\left(\sqrt{(n+\lambda) P_{k-1}}\right)_{h-n} \mathrm{~h}=n+1, \cdots, 2 n
\end{array}\right. \\
& \left\{\begin{array}{l}
W_{0}^{m}=\lambda /(n+\lambda) \\
W_{0}^{c}=\lambda /(n+\lambda)+\left(1-\alpha^{2}+\beta\right) \\
W_{h}^{m}=W_{h}^{c}=\lambda / 2(n+\lambda) \quad \mathrm{h}=1, \cdots, 2 n
\end{array}\right.
\end{aligned}
$$

Where $\left(\sqrt{(n+\kappa) P_{k-1}}\right)_{h}$ is obtained through Cholesky decomposition Parameter $\lambda$ is.

$$
\lambda=\alpha^{2}(n+\kappa)-n
$$

\section{Modeling Target State Estimation based on Interacting Multiple Model-Unscented Filter in RNS}

Based on property of RNS, this paper intends to use three models to construct interacting multiple model group, namely uniform constant velocity (CV) model, anticlockwise collaborative turn (ACT) model and clockwise collaborative turn $(\mathrm{CCT})$ model.

\footnotetext{
${ }^{*}$ The National Nature Science Fund Project 61273001, Anhui Province Nature Science Fund Project 11040606 M130
} 


\subsection{Modeling state/observation}

Assuming state and measurement noises are additive white Gaussian noise, the state and measurement equations for IMM-UFA are as formula (7) and (8) below.

$$
\begin{aligned}
& X_{k}=\Phi_{k / k-1} X_{k-1}+\Gamma_{k / k-1} W_{k-1} \\
& Z_{k}=h\left(X_{k}\right)+V_{k}
\end{aligned}
$$

Where $X_{k}=\left[\begin{array}{llll}x_{k}^{f} & \dot{x}_{k}^{f} & y_{k}^{f} & \dot{y}_{k}^{f}\end{array}\right]^{T}$ and $Z_{k}=\left[\begin{array}{lll}r_{k} & \theta_{k} & \varphi_{k}\end{array}\right]^{T}$. The statistical covariance of $V_{k}$ is $R_{k}$. Let $Q_{k-1}$ be covariance of that instigated white noise.

\subsection{Modeling interactive input}

When IMM-UFA is used to for target state estimate in RNS, it is needed to recalculate the initial state and covariance estimation for each model.

$$
\begin{gathered}
\hat{X}_{k-1}^{0 j}=\sum_{i=1}^{r} \hat{X}_{k-1}^{i} \mu_{k-1}^{i / j} \\
P_{k-1}^{0 j}=\sum_{i=1}^{r} \mu_{k-1}^{i / j}\left\{P_{k-1}^{i}+\left[\hat{X}_{k-1}^{i}-\hat{X}_{k-1}^{0 j}\right]\left[\hat{X}_{k-1}^{i}-\hat{X}_{k-1}^{0 j}\right]^{T}\right\}
\end{gathered}
$$

Where $\mathrm{r}$ is the number of models in model group. $\hat{X}_{k-1}^{i}$ and $P_{k-1}^{i}$ are state estimation and covariance at timestep k-1 for member i. $\mu_{t+1}^{i \prime j}$ is model mixture probability. Calculating $\mu_{k-1}^{i / j}$

$$
\mu_{k-1}^{i / j}=P\left\{M_{k-1}=i / M_{k-1}=j, Z^{k-1}\right\}=\frac{p_{i / j} \mu_{k-1}^{i}}{\sum_{i=1}^{n} p_{i / j} \mu_{k-1}^{i}}
$$

Where $\mu_{k-1}^{i}$ is model probability at the previous timestep. $p_{i / j}$ is the inherent Markov transition probability of model group.

Initial state estimation $\hat{X}_{0}^{i}$ and covariance matrix $P_{0}^{i}$ are needed in the initial interaction of model members. Since this paper only estimate position and speed of air maneuvering target in XY plane of fusion center coordinate system, therefore, this paper uses two-point difference method to calculate initial state estimation $\hat{X}_{0}^{i}$ and covariance $P_{0}^{i}$, making use of the measurement data at first and second timestep to calculate.

Let $Z_{1^{\prime}}=\left[z_{x_{1}}, z_{y_{1}^{\prime}}\right]^{T}$ and $Z_{2^{\prime}}=\left[z_{x_{2}}, z_{y_{2}}\right]^{T}$ be the first and second measurement points of target initial track (denoted as $1^{\prime}$ and $2^{\prime}$ track point), which are coordinate transformation measured value of airspace maneuvering target in fusion center inertial coordinate system (referred to transformation measured value). Therefore, based on measurement of the first two points, the initial state estimation is as the formula (12) below.

$$
\hat{X}_{0}^{i}=\left[\begin{array}{llll}
z_{x_{i}} & \frac{z_{x_{z}}-z_{x_{i}}}{T} & z_{y_{y_{i}}} & \frac{z_{y_{z}}-z_{y_{Y}}}{T}
\end{array}\right]^{T}
$$

Let $X_{1^{\prime}}=\left[\begin{array}{llll}x_{1^{\prime}}^{f} & \dot{x}_{1^{\prime}}^{f} & y_{1^{\prime}}^{f} & \dot{y}_{1^{\prime}}^{f}\end{array}\right]^{T}$ and $X_{2^{\prime}}=\left[\begin{array}{llll}x_{2^{\prime}}^{f} & \dot{x}_{2^{\prime}}^{f} & y_{2^{\prime}}^{f} & \dot{y}_{2^{\prime}}^{f}\end{array}\right]^{T}$ be true target state for the point $1^{\prime}$ and $2^{\prime}$. Computing relationship between true state and transformation measured value.

$$
\begin{aligned}
& \left\{\begin{array}{l}
x_{1^{\prime}}^{f}=z_{x_{1^{\prime}}}+\Delta x_{1^{\prime}}^{f} \\
y_{1^{\prime}}^{f}=z_{y_{1^{\prime}}}+\Delta y_{1^{\prime}}^{f}
\end{array}\right. \\
& \left\{\begin{array}{l}
x_{2^{\prime}}^{f}=z_{x_{2^{\prime}}}+\Delta x_{2^{\prime}}^{f} \\
y_{2^{\prime}}^{f}=z_{y_{2^{\prime}}}+\Delta y_{2^{\prime}}^{f}
\end{array}\right.
\end{aligned}
$$

Wher $\left[\begin{array}{ll}\Delta x_{1^{\prime}}^{f} & \Delta y_{1^{\prime}}^{f}\end{array}\right]^{T}$ and $\left[\begin{array}{ll}\Delta x_{2^{\prime}}^{f} & \Delta y_{2^{\prime}}^{f}\end{array}\right]^{T}$ are errors between transformation measured value and true state of point $1^{\prime}$ and $2^{\prime}$.

Based on transformation measured value of the first two timestep, calculating the error of initial state estimation and the second order central moment of initial estimation error

$$
X_{0}^{i}-\hat{X}_{0}^{i}=\left[\begin{array}{c}
\Delta x_{2^{\prime}}^{f} \\
\frac{\Delta x_{2^{\prime}}^{f}-\Delta x_{1^{\prime}}^{f}}{T} \\
\Delta y_{2^{\prime}}^{f} \\
\frac{\Delta y_{2^{\prime}}^{f}-\Delta y_{1^{\prime}}^{f}}{T}
\end{array}\right]
$$

$$
\begin{aligned}
& P_{\theta}^{*}=E\left[\left(X_{\theta}^{*}-\hat{X}_{\theta}^{*}\right)\left(X_{\theta}^{*}-\hat{X}_{\theta}^{*}\right)^{*}\right]=
\end{aligned}
$$

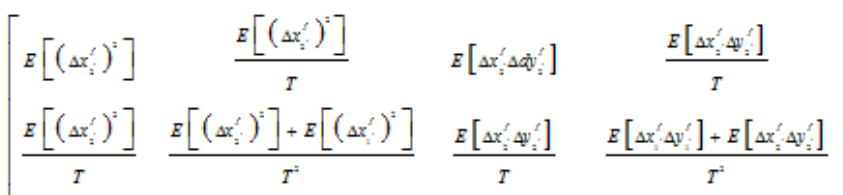

$$
\begin{aligned}
& E\left[\Delta x_{i}^{\prime} \Delta y_{i}^{\prime}\right] \quad \frac{E\left[\Delta x_{i}^{\prime} \Delta y_{i}^{t}\right]}{T} \quad E\left[\left(\Delta y_{i}^{\prime}\right)^{i}\right] \quad \frac{E\left[\left(\Delta y_{i}^{f}\right)^{i}\right]}{T}
\end{aligned}
$$

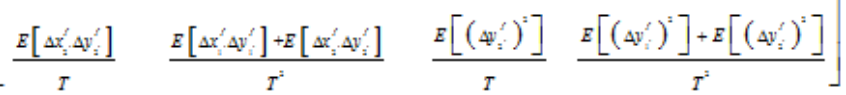

Where $E\left[\left(\Delta x_{1^{\prime}}^{f}\right)^{2}\right], \quad E\left[\left(\Delta x_{z^{\prime}}^{\prime}\right)^{2}\right], \quad E\left[\left(\Delta y_{1^{\prime}}^{\prime}\right)^{2}\right], \quad E\left[\left(\Delta y_{2^{\prime}}^{\prime}\right)^{2}\right]$ are covariance of errors of point $1^{\prime}$ and $2^{\prime}$ in $\mathrm{x}$, y direction of fusion center inertial coordinate system. The errors are caused by measurement noise in NR. $E\left[\Delta x_{1^{\prime}}^{f} \Delta y_{1^{\prime}}^{f}\right]$ and $E\left[\Delta x_{2^{\prime}}^{f} \Delta y_{2^{\prime}}^{f}\right]$ are x-y cross-covariances (formula [9]).

\subsection{Modeling state prediction}

Generating predicted $\sigma$ point set and state prediction statistics.

$$
\begin{aligned}
& \chi_{k \mid k-1}^{h}=\Phi_{k \mid k-1}^{j} \chi_{k-1}^{h}+\Gamma_{k \mid k-1}^{j} W_{k-1}^{h} \\
& \hat{X}_{k \mid k-1}^{j}=\sum_{h=0}^{2 n} W_{h}^{m} \chi_{k \mid k-1}^{h}
\end{aligned}
$$




$$
\begin{aligned}
P_{k \mid k-1}^{j}= & \sum_{k=0}^{2 \pi}\left\{W_{k}^{c}\left(\chi_{k \mid k-1}^{k}-\hat{X}_{k \mid k-1}^{j}\right)\left(\chi_{k \mid k-1}^{k}-\hat{X}_{k \mid k-1}^{j}\right)^{T}\right\} \\
& +\Gamma_{k-1} Q_{k-1} \Gamma_{k-1}^{T}
\end{aligned}
$$

\subsection{Modeling observation prediction}

Generate the predictive $\sigma$ point set, observation $\sigma$ point set, the statistics of observation $\sigma$ point set and state prediction cross-covariance matrix.

$$
\begin{gathered}
\mathfrak{J}_{k / k-1}^{h}=h\left(\chi_{k / k-1}^{h}\right)+V_{k}^{h} \\
\hat{Z}_{k / k-1}^{j}=\sum_{h=0}^{2 n} W_{h}^{m} \Im_{k / k-1}^{h} \\
P_{k / k-1}^{Z}=\sum_{h=0}^{2 n} W_{n}^{c}\left\{\left(\mathfrak{\Im}_{k / k-1}^{h}-\hat{Z}_{k / k-1}^{j}\right)\left(\mathfrak{\Im}_{k / k-1}^{h}-\hat{Z}_{k / k-1}^{j}\right)^{T}\right\}+R_{k}(22) \\
P_{k / k-1}^{X Z}=\sum_{h=0}^{2 n} W_{h}^{c}\left\{\left(\chi_{k / k-1}^{h}-\hat{X}_{k / k-1}^{j}\right)\left(\mathfrak{\Im}_{k / k-1}^{h}-\hat{Z}_{k / k-1}^{j}\right)^{T}\right\}
\end{gathered}
$$

\subsection{Modeling state estimation}

Based on the linear minimum covariance estimation as equation (1) shown, calculating state estimation $\hat{X}_{k}^{j}$ and its covariance matrix $P_{k}^{j}$ for model $\mathrm{j}$

$$
\begin{aligned}
& \hat{X}_{k}^{j}=\hat{X}_{k / k-1}^{j}+P_{k / k-1}^{X Z}\left(P_{k / k-1}^{Z}\right)^{-1}\left(Z_{k}-\hat{Z}_{k / k-1}^{j}\right) \\
& P_{k}^{j}=P_{k / k-1}^{j}-P_{k / k-1}^{X Z}\left(P_{k / k-1}^{Z}\right)^{-1} P_{k / k-1}^{X Z}
\end{aligned}
$$

\subsection{Modeling model probability}

Based on Bayes rule, model probability can be expressed as formula (26) below.

$$
\begin{aligned}
& \mu_{k}^{j}=P\left\{M_{k}=j / Z^{k}\right\}= \\
& \frac{P\left\{Z_{k} / M_{k}=j, Z^{k-1}\right\} P\left\{M_{k}=j / Z^{k-1}\right\}}{P\left\{Z^{k}\right\}}
\end{aligned}
$$

Where $P\left\{Z_{k} / M_{k}=j, Z^{k-1}\right\}$ is the estimation likelihood function of model j. $P\left\{M_{k}=j / Z^{k-1}\right\}$ is the selection probability of prediction model $\mathrm{j}$ at timestep k-1 (prediction model probability ). $P\left\{z^{*}\right\}$ is a normalization factor. Let $\Lambda_{k}^{j}$ be estimation likelihood function $P\left\{Z_{k} / M_{k}=j, Z^{k-1}\right\}$

$$
\begin{aligned}
& \Lambda_{k}^{j}=P\left\{Z_{k} / M_{k}=j, Z^{k-1}\right\}= \\
& \frac{1}{\sqrt{(2 \pi)^{3}\left|P_{k / k-1}^{Z}\right|}} \exp \left\{-\frac{1}{2} v_{j}^{T}\left(P_{k / k-1}^{Z}\right)^{-1} v_{j}\right\} \\
& P\left\{M_{k}=j / Z^{k-1}\right\}=\sum_{i=1}^{r} p_{i / j} \mu_{k-1}^{i}
\end{aligned}
$$

$$
\mu_{k}^{j}=\frac{\Lambda_{k}^{j} \sum_{i=1}^{r} p_{i / j} \mu_{k-1}^{i}}{\sum_{j=1}^{r} \Lambda_{k}^{j} \sum_{i=1}^{r} p_{i / j} \mu_{k-1}^{i}}
$$

\subsection{Modeling interactive state estimation output}

The final state estimation of the target is based on the probability-weighted output of all members.

$$
\begin{gathered}
\hat{X}_{z}=\sum_{j=-1}^{\infty} \hat{X}_{z}^{\prime} \mu_{z}^{\prime} \mid \\
P_{.}=\sum_{j=1} \mu_{.}^{j}\left\{P_{.}^{j}+\left[\hat{X}_{.}^{j}-\hat{X}_{.}\right]\left[\hat{X}_{.}^{j}-\hat{X}_{.}\right]^{\tau}\right\}
\end{gathered}
$$

\section{Simulation Verification and Conclusion Analysis}

In order to verify the effectiveness and practicality of the algorithm above, we use the software simulation method to test. Simulation scenario is as follows: two networked radars (radar 1 and radar 2); scanning period of them is 10 seconds; position of radar 1 is $\left[\begin{array}{lll}118^{\circ} & 29^{\circ} & 120\end{array}\right]^{\mathrm{T}}$; rang, azimuth, and pith angle measurement accuracy of radar 1 is $150 \mathrm{~m}, 0.3^{\circ}$, and

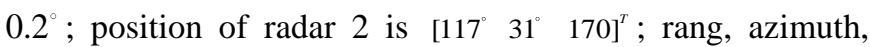
and pith angle measurement accuracy of radar 2 is $100 \mathrm{~m}$,

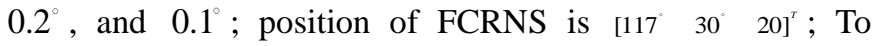
short-cut calculation, let detecting time interval between two radars is always $5 \mathrm{~s}$ in simulation. There are 300 detect point marks in all, detecting one sampling point at every $5 \mathrm{~s}$. Model group includes $\mathrm{CV}, \mathrm{ACT}$ and $\mathrm{CCT}$ model. First, the target motions in CV model at speed of $200 \mathrm{~m} / \mathrm{s}$ for $500 \mathrm{~s}$. Second, the target motions in ACT model at angular velocity of $0.007 \mathrm{rad} / \mathrm{s}$ for $500 \mathrm{~s}$. Third, the target motions in CCT model at angular velocity of $-0.007 \mathrm{rad} / \mathrm{s}$ for $500 \mathrm{~s}$. Entire flight altitude is $6000 \mathrm{~m}$. Let $\sigma_{x}=0.8, \sigma_{y}=0.8$, Markov transition probability $\quad P_{i / j} \quad$ be $\left[\begin{array}{ccc}0.95+0.05 / 3 & 0.05 / 3 & 0.05 / 3 \\ 0.05 / 3 & 0.95+0.05 / 3 & 0.05 / 3 \\ 0.05 / 3 & 0.05 / 3 & 0.95+0.05 / 3\end{array}\right]$, initial model probability be $\left[\begin{array}{llll}0.95+0.05 / 3 & 0.05 / 3 & 0.05 / 3\end{array}\right]^{T}$. Repeat the simulation 100 times.

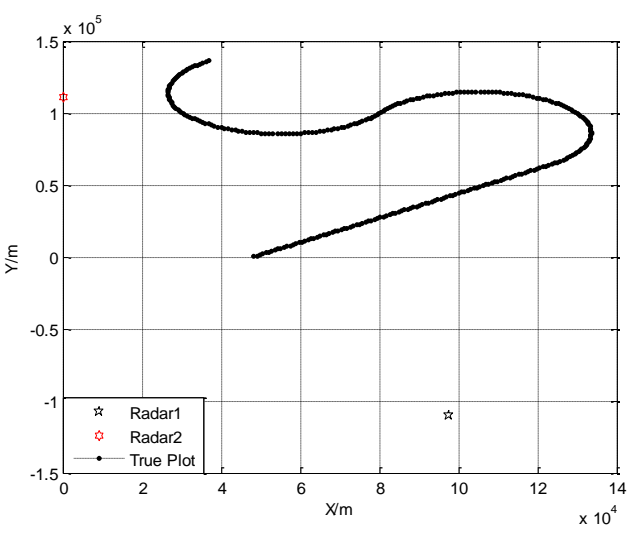

Figure 1 RNS simulation scenario 


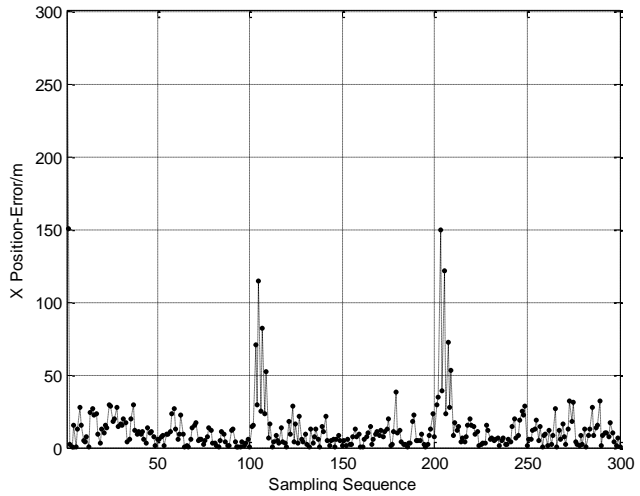

Figure 2 IMM-UFA position estimation errors(X)

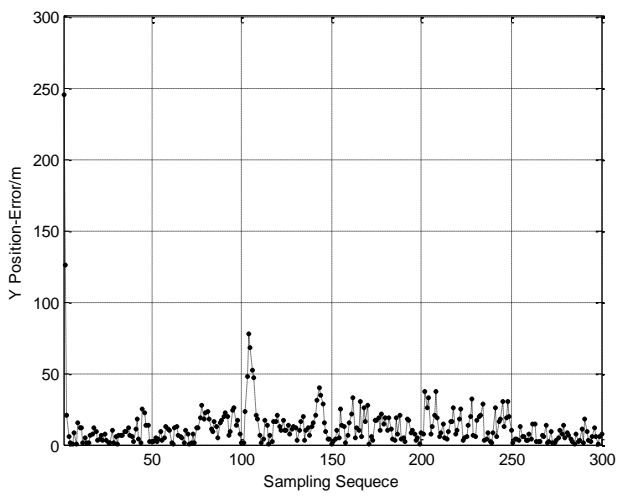

Figure 3 IMM-UFA position estimation errors(Y)

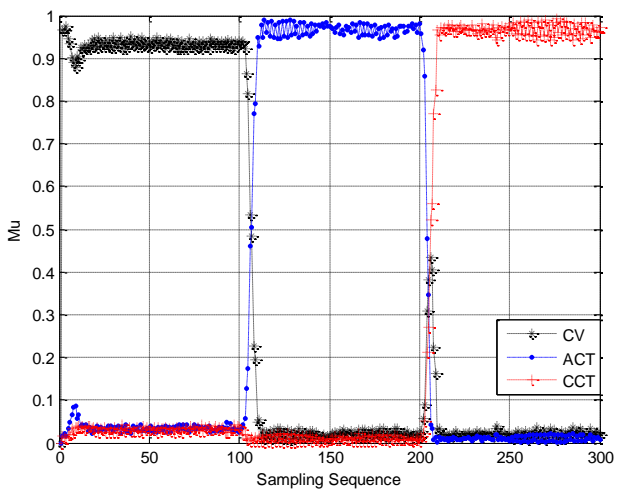

Figure 4 changes of model probability
Figure 1 shows simulation scenarios of two networked radars. Figure 2 and 3 shows errors of IMM-UFA position estimation of 100 simulation. According to figure 2 and 3, initialization strategy proposed in this paper has good astringency when it is used in IMM-UFA, creating no initial large fluctuations. Because of measurement noise of NR, measurement points have large fluctuations when compared with true points. Estimation points of constructed-parameter IMM-UFA proposed in this paper can well approximate true points, with small estimation error. But in the maneuver corner, the estimation errors have some ups and downs. A few frames later, the estimation stabilizes. Figure 4 shows changes of model probability in each stage of target motion. It can be seen that IMM-UFA can adaptively approximate true motion model. Simulation results show that the model in IMM-UFA used in this paper is consistent with true maneuvering target model.

\section{References}

[1] Zhang Wei, Ma Hui-ping, Wang Yong-hai, Kang Peng. A study on the influence between station distribution of netted radar system and data fusion accuracy, Modem Radar. 32(7):11-15, 2010.

[2] B1om.H.A, Bar-Shalom.Y. The Interacting Multiple Model Algorithm for Systems with Markovian Switching Coefficients. IEEE Trans. on Automatic Control, 1988, 33(8):780-783.

[3] Liao Yonghan, Zhu Shengli, Peng Dongliang. IMM filter with application to bearings-only passive maneuvering target tracking. Fire Control \& Command Control. Jan, 2010, 35(1):20-23.

[4] Kalman.R.E. A new approach to linear filter and prediction problems. Transactions of the ASME-Journal of Basic Enginearing. 1960,82:34-45.

[5] Kai Xiong, Tang Lianga, Lei Yongjuna. Multiple model Kalman filter for attitude determination of precision pointing spacecraft. Acta Astronauautica, April-May 2011, 68(7):843-852.

[6] Simon J. Julier. The scaled unscented transformation. Proceedings of the American Control Conference. Jefferson City, May 8-10, 2002, 4555-4559.

[7] Orlando X. Diaz. Analysis and Comparison of Extended and Unscented Kalman Filtering Methods for Spacecraft Attitude Determination. Master Thesis of Naval Postgraduate School, December 2010.

[8] Qing Yong-yuan, Zhang Hong-yue, Wang Shu-hua, Kalman filter and navigation principle. Xian: Northwestern Polytechnical University Press .1998, 11, 1-43.

[9] Zhao Wen-bo, Du Ji-yan. Study on statistics of errors of noise in inertial coordinate system, Journal of Artillery Academy, 2010, 05, 91-95. 